\title{
800 MHZ LOW MOTIONAL RESISTANCE CONTOUR-EXTENSIONAL ALUMINUM NITRIDE MICROMECHANICAL RESONATORS
}

\author{
Philip J. Stephanou and Albert P. Pisano \\ Berkeley Sensor \& Actuator Center (BSAC) \\ The University of California at Berkeley, Berkeley, CA 94720
}

\begin{abstract}
This work introduces novel contour-extensional mode piezoelectric Aluminum Nitride micro-mechanical resonators that enable breakthrough increases in operating frequency and reductions in motional resistance. By selectively patterning the transduction electrodes and routing the excitation waveform, the resonant frequency of the device is effectively uncoupled from the overall dimensions of the AlN plate. Consequently, the motional resistance of a resonator with a given frequency can be significantly reduced by scaling up the lateral dimensions of the structural layer. The design has been validated by demonstrating an $803 \mathrm{MHz}$ resonator with $24 \Omega$ motional resistance and a $Q$ factor over 1,000 when tested in air.
\end{abstract}

\section{INTRODUCTION}

The demand for highly-integrated analog filtering and frequency reference elements has spurred rapid innovation in the area of vibrating RF MEMS [1]. To date however, no single technology has emerged that can simultaneously deliver monolithic, post-CMOS integration of IF and RF components that can readily interface with $50 \Omega \mathrm{RF}$ systems. Thickness-extensional FBAR resonators have proven the technical feasibility and commercial viability of thin film piezoelectric Aluminum Nitride (AlN) based processing technology for RF applications, but the designs are practically limited to a single frequency per AlN deposition and not scaleable to IF [2]. Air and solid dielectric gap electrostatic contour mode resonators inherently do not suffer either of the aforementioned limitations, but despite monumental improvements have yet to demonstrate low motional resistance, fundamental mode resonators suitable for RF filter synthesis $[\mathbf{3 , 4}]$. AlN contour mode MEMS resonators have emerged as the premier technology for realizing multi-frequency per chip, CMOScompatible, low-loss filters, but heretofore have been limited to operating frequencies between 10 and $500 \mathrm{MHz}[\mathbf{5 , 6 ]}$.

The present work introduces a novel design for piezoelectric contour mode MEMS plate resonators that effectively uncouples the resonant frequency of the devices from their overall dimensions by selectively patterning the transduction electrodes and routing the excitation waveform as seen in Figure 1. The ability to scale the lateral dimensions of the structural material enables more mechanically robust devices that are capable of attaining higher frequencies ( $803 \mathrm{MHz}$ demonstrated) with reduced motional resistances $(24 \Omega$ ) and relaxed fabrication tolerances (structural plate measures $51 \times 100 \mu \mathrm{m}$ ). The electrode configuration also suppresses the appearance of any lower or subharmonic modes in the frequency response of the device.

\section{THEORY}

Piezoelectric and electrostatic contour mode MEMS resonators based on rectangular or annular plates offer the ability to prescribe frequency and motional resistance independently within a limited design space, but are ultimately precluded from reaching $\mathrm{GHz}$ fundamental modes by the need to define halfwavelength features (on the order of several microns) in whatever structural material is being used [3,7]. Theoretical designs for low motional resistance, $\mathrm{GHz}$ rectangular or annular plate resonators are marked by extreme length to width or average radius to annular width, respectively, which in practice result in unacceptable mechanical compliance, inefficient use of layout area, and the need for exacting fabrication tolerances.

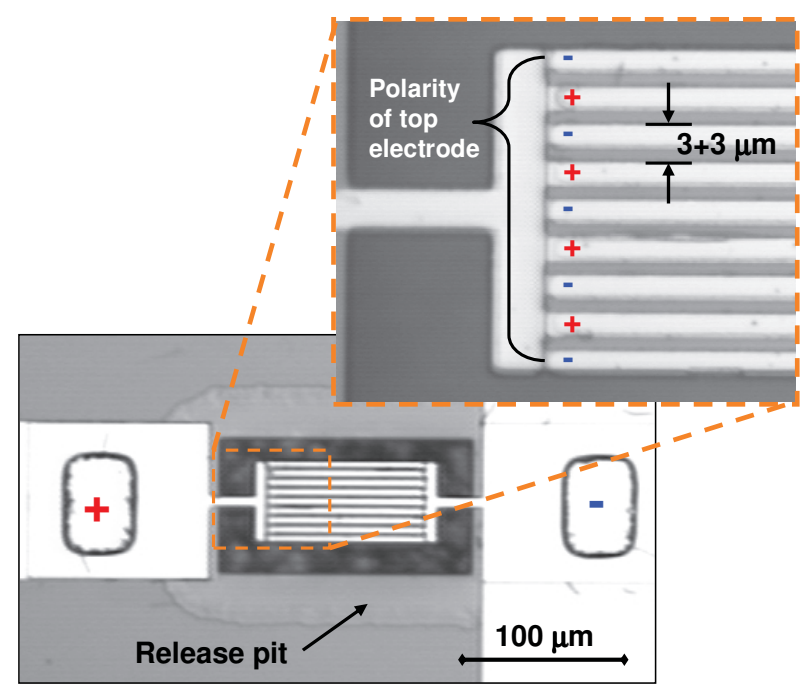

Figure 1: Optical micrograph of micromechanical resonator with selectively patterned half-wavelength period electrodes. The polarity of the electric excitation signal alternates between adjacent top and bottom electrode pairs. The effective characteristic lengths for determining frequency and motional resistance are 6 and $900 \mu m$, respectively.

In the present design, the frequency determining dimension is effectively uncoupled from the overall dimensions of the AlN plate by patterning the transduction electrodes such that the polarity of the excitation electric field alternates at half-wavelength intervals in the direction of wave propagation as seen in Figure 2. The polarity of the electrodes uniquely matches that of the strain field for a specific bulk mode of vibration of the plate. Moreover, the number of half-wavelength electrodes can be scaled to engineer a proportional decrease in motional resistance (the motional resistance also depends on the length of the plate).

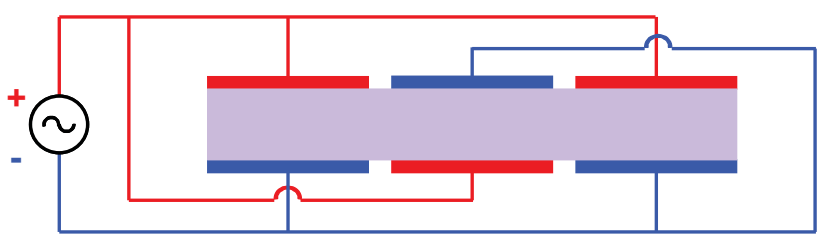

Figure 2: Schematic of resonator cross-section showing selective electrode patterning and signal routing. The polarity of each top and bottom electrode pair alternates at half-wavelength intervals. The period of the electrode pairs determines the operating frequency of the resonator while the number of periods affects its motional resistance. 
The practical implication of the design is that half-wavelength features need only be defined in the thin metal electrodes, and the feasibility of fabricating such features in a production environment has essentially been proven by SAW device manufacturers (in fact SAW devices have more demanding fabrication requirements due to their lower acoustic wavespeed). As with SAW devices, the nominal frequency of the resonator depends not only on the absolute dimensions but also the periodicity of the electrodes.

\section{FABRICATION PROCESS}

The resonators under investigation are fabricated using a variation of a previously published four-mask, low-temperature process [7]. The device consists of a thin film piezoelectric AlN structural layer sandwiched between Pt bottom and top electrodes. The approximate thicknesses of the AlN and Pt layers are $1.5 \mu \mathrm{m}$ and $200 \mathrm{~nm}$, respectively. A $300 \mathrm{~nm}$ low-stress silicon nitride layer is deposited between the silicon substrate and bottom electrode for electrical isolation. The AIN films are deposited using a singlemodule AMS Inc. sputtering tool.

\section{EXPERIMENTAL DETAILS}

The fabricated MEMS filters are tested in a Janis RF probe station with micro-manipulated ground-signal-ground (GSG) probes. All testing is performed in air at atmospheric pressure and ambient temperature. The $S_{11}$ parameter of the devices is extracted by an Agilent E5071B vector network analyzer with $0 \mathrm{dBm}$ of signal power following a short-open-load calibration on a ceramic reference substrate. The admittance transformation is calculated by the network analyzer.

Figure 3 shows a plot of the admittance response of a piezoelectric contour mode MEMS resonator over a 200 to $1,000 \mathrm{MHz}$ frequency range. The AlN plate in this instance measures $51 \times 100 \mu \mathrm{m}$ and has 9 pairs of Pt top and bottom electrodes with $3 \mu \mathrm{m}$ line width and spacing. Notice the effective suppression of spurious modes achieved by the electrode configuration.

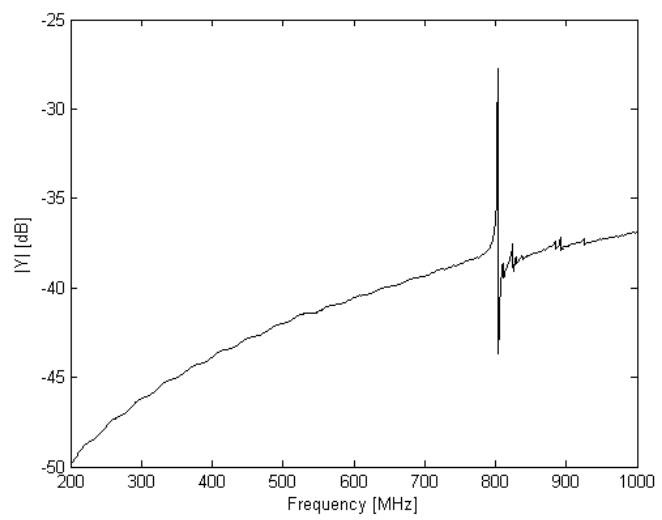

Figure 3: Admittance response of a micromechanical resonator with 9 half-wavelength top and bottom electrode pairs showing effective suppression of spurious modes from $200 \mathrm{MHz}$ to $1 \mathrm{GHz}$.

Figure 4 shows the admittance response of the same resonator in the vicinity of its $803 \mathrm{MHz}$ series resonance exhibiting $24 \Omega$ motional resistance and a $Q$ factor of 1,300 when tested in air. A similar resonator with 7 pairs of $3 \mu \mathrm{m}$ line and space electrodes displayed an $828 \mathrm{MHz}$ resonant frequency with $58 \Omega$ motional resistance and a $Q$ of 1,000 when tested in air.

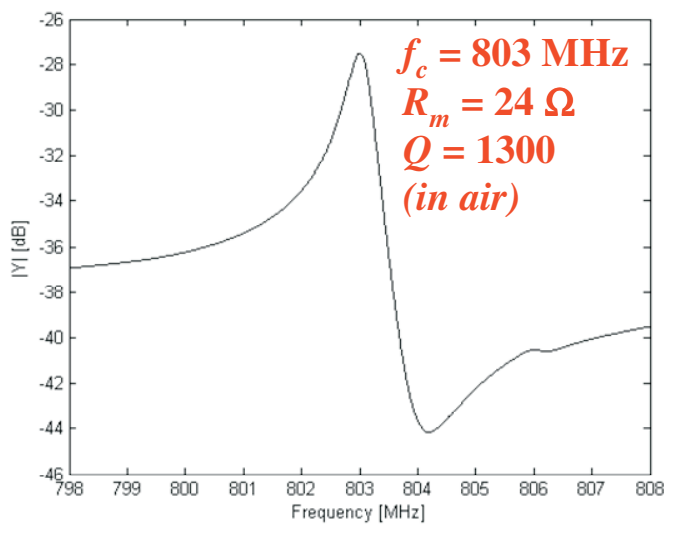

Figure 4: Admittance response of same resonator as Figure 3 showing resonant behavior. Testing is done in air with $0 \mathrm{dBm}$ signal power. Data are measured directly on network analyzer.

\section{CONCLUSIONS}

Novel piezoelectric contour mode MEMS resonators have been demonstrated that enable breakthrough increases in frequency and reductions in motional resistance. The performance gains stem from a technique for selectively patterning the transduction electrodes and routing the excitation waveform. An AlN resonator measuring $51 \times 100 \mu \mathrm{m}$ overall with 9 pairs of $3 \mu \mathrm{m}$ line and space Pt electrodes has been tested at $803 \mathrm{MHz}$ with $24 \Omega$ motional resistance and a $Q$ of 1,300 in air. This technology promises to for the first time permit monolithic integration of post-CMOS compatible, low-loss filters spanning IF to RF that can readily be interfaced with existing $50 \Omega$ RF systems.

\section{ACKNOWLEDGEMENTS}

This work was supported by DARPA grant No. NBCH1020005. The authors offer special thanks to AMS Inc. for their assistance with AlN deposition and tool installation, and to the UC Berkeley Microfabrication Laboratory staff for their support.

\section{REFERENCES}

[1] K.M. Lakin et al., "Development of Miniature Filters for Wireless Applications," IEEE Trans. on Microwave Theory and Tech., vol. 43, no. 12, pp. 2933-2939, 1995.

[2] R. Ruby, et al., Y. Oshmyansky, "PCS $1900 \mathrm{MHz}$ duplexer using thin film bulk acoustic resonators (FBARs)," Electronics Lett. 35, pp. 794, 1999.

[3] J. Wang, C.T.-C. Nguyen, et al., "1.156-GHz Self-Aligned Vibrating Micromechanical Disk Resonator," IEEE Trans. on UFFC, vol. 51, no. 12, pp. 1607-1628, 2004.

[4] Y.-W. Lin, .T.-C. Nguyen, et al., "Vibrating micromechanical resonators with solid dielectric capacitive transducer gaps," IEEE Proc. Freq. Cont. Symposium and Exhibition, pp. 128-134, 2005.

[5] G. Piazza, et al., "Single-chip multiple-frequency filters based on contour-mode aluminum nitride piezoelectric micromechanical resonators," TRANSDUCERS 2005, pp. 2065-2068, 2005.

[6] P.J. Stephanou, et al., "Mechanically Coupled Contour Mode Piezoelectric Aluminum Nitride MEMS Filters," IEEE MEMS 2006, pp. 906-909, 2006.

[7] G. Piazza, et al., "Low motional resistance ring-shaped contour-mode aluminum nitride piezoelectric micromechanical resonators for UHF applications," IEEE MEMS 2005, pp.20-3, 2005. 